\title{
Pre- and post-operative differences between genders in idiopathic macular holes
}

\author{
Jing Wang ${ }^{\dagger}$, Yanping Yu ${ }^{\dagger}$, Xida Liang, Zengyi Wang, Biying Qi and Wu Liu* (D)
}

\begin{abstract}
Background: To compare idiopathic macular holes (IMHs) between male and female before and after surgery.

Methods: Patients with IMHs of stage 3 and stage 4 who underwent 23-gauge vitrectomy were retrospectively enrolled. Pre-operative clinical features like age of onset, and best-corrected visual acuity (BCVA) were reviewed. Optical coherence tomography parameters including minimum linear diameter (MLD), central macular thickness and some other indexes were measured and calculated. Main surgical outcomes included the primary closure rate, the highest BCVA during follow-up, and the recovery duration. All the metrics mentioned above were compared between genders with appropriate statistical methods.

Results: A total of 298 eyes from 280 patients (male: 51; female: 229) were enrolled. Compared with men, women demonstrated a significantly higher ratio of stage3/stage4 $(P=0.045)$, larger horizontal MLD $(P=0.009)$, but similar surgical outcomes except for a relatively longer recovery duration $(P=0.024)$. For stage $3 \mathrm{IMHs}$, women exhibited significantly younger age of onset $(P=0.023)$, larger MLD $(P=0.003)$, and smaller height of the hole $(P=0.029)$. However, for stage $4 \mathrm{IMHs}$, all the pre- and post-operative metrics showed no differences between genders.

Conclusions: Female IMHs seem to demonstrate an earlier age of onset and larger size of hole, especially in IMHs of stage 3. However, these differences, which may owe to normal gender-related variations, have limited influence on the surgical outcomes.
\end{abstract}

Keywords: Gender variation, Idiopathic macular hole, Optical coherence tomography, Vitrectomy

\section{Background}

Idiopathic macular holes (IMHs) are full-thickness defect in the neuroretina with unknown specific causes [1], which greatly reduce vision health-related quality of life [2]. The incidence of IMH is approximately 0.01 to $0.09 \%$ [3], with a female to male ratio of about $3: 1$ [3]. Although the pathogenesis of IMH is not completely clear, vitreomacular traction has been considered as one of the most important motivating factors [4].

\footnotetext{
* Correspondence: wuliubj@sina.com

${ }^{\dagger}$ Jing Wang and Yanping Yu contributed equally to this work.

Beijing Tongren Eye Center, Beijing Tongren Hospital, Capital Medical University; Beijing Ophthalmology and Visual Sciences Key Laboratory, No 1, Dongjiaominxiang Street, Dongcheng District, Beijing 100730, China
}

Clinically, gender variation is not rare in fundus diseases. Several studies considered male gender as a risk factor for central serous chorioretinopathy (CSC) [5], and female subjects with CSC tend to obtain better prognosis [6]. Another report showed that women had a potentially higher risk of neovascular age-related macular degeneration compared to men [7]. Besides different incidences, male and female IMHs may exhibit different manifestations and surgical outcomes since women demonstrate wider foveal pit and thinner central retina [8]. However, few previous studies have focused on this issue. 
This study aims to compare pre-operative clinical features, morphological characteristics (especially OCT parameters) and surgical outcomes of stage 3 and stage 4 IMHs between genders based on a relatively large sample, and try to find differences of IMH between genders so as to provide reference for clinical applications.

\section{Methods}

\section{Patients and examinations}

Patients with IMHs of stage 3 or stage 4 who underwent 23-gauge vitrectomy in Beijing Tongren Hospital from July 2015 to August 2018 were retrospectively enrolled. The exclusion criteria were as follows: stage 2 macular holes, traumatic macular holes, history of uveitis or other fundus diseases, and history of intravitreal injection, vitreous surgery or retinal detachment surgery. This study adhered to the tenets of the Declaration of Helsinki and was approved by the Ethics Committee of Beijing Tongren Hospital. Informed consent was obtained from all the subjects after explanation of the nature and possible consequences of the study.

Clinical charts of all patients were reviewed in detail, including date of birth (A), gender, affected eye, duration of symptoms (B), date of operation (C), intraocular pressure (IOP), axial length (AXL), and best-corrected visual acuity (BCVA). IOP came from noncontact tonometry (Full Auto Tonometer TX-F; Canon Canada, Quebec), and AXL was measured by IOL Master Biometry (Carl Zeiss Meditec, Jena, Germany). Every participant underwent slit-lamp microscopy examination for the anterior segment, and comprehensive fundus examinations were carried out by binocular indirect ophthalmoscope and fundus photography (fundus camera, TRC-50; Topcon, Tokyo, Japan). The diagnosis of IMH was based on clinical features, spectrum domain OCT (Cirrus high definition OCT; Carl Zeiss, Dublin, CA) imaging, fundus examination and intraoperative observation. According to Gass's classification of 1995 [9], stages of IMH were identified by OCT and intraoperative observations, the majority of which were considered as large IMHs based on the 2013 consensus [10]. Age of onset was defined as the time when symptoms occurred and calculated by (CA)-B. The recovery duration was the interval between the primary surgery and the occurrence of the highest BCVA during follow-up.

\section{OCT measurements}

OCT examination was performed by the same experienced operator, and every measurement was repeated three times for an average to reduce manual errors. The entire macula was scanned by Macular Cube $512 \times 128$ protocol in horizontal and vertical orientations. The minimum linear diameter (MLD) was defined as the shortest distance between the broken ends of the neuroepithelia parallel to the retinal pigment epithelium (RPE). The basal diameter (BD) was the length of the RPE where the photoreceptors were detached, and $\mathrm{H}$ was the average vertical height on both sides, from the junction point of detached photoreceptor and RPE to the top of neuroepithelium. The fluid cuff (FC) was calculated by BD-MLD, diameter hole index (DHI) was MLD/BD, macular hole index (MHI) was $\mathrm{H} / \mathrm{BD}$, traction hole index (THI) was H/MLD, and hole form factor (HFF) was (MLD + $\mathrm{BD}) / \mathrm{BD}$.

Central macular thickness (CMT) was the automatic average thickness of foveal retina in a circular area with a diameter of $1 \mathrm{~mm}$. The CMT of the affected eyes was undetectable, so CMT of the normal fellow eyes was measured to represent. Fellow eyes with normal CMT (within normal range of $240 \pm 20 \mu \mathrm{m}$ [11]), no history of fundus diseases or vitreous surgery and $\mathrm{BCVA} \geq 0.5$ were included. Adjusted CMT was calculated by CMT/AXL.

\section{Surgical procedure}

All the patients enrolled underwent standard 3-port pars plana vitrectomy by the same experienced surgeon using the 23-gauge technique. Phacoemulsification and intraocular lens implantation were combined prior to vitrectomy if necessary. After the core vitrectomy with the trocars inserted obliquely, the posterior cortical vitreous was detached (in stage $3 \mathrm{IMHs}$ ) and the vitreous was meticulously cut to the vitreous base. Then the internal limiting membrane (ILM) was peeled with intraocular forceps over a circle with a diameter of 2 to 3 optic discs centered in the macular hole without prior staining. Any epiretinal membranes would have been peeled before ILM peeling if presented. Afterwards, fluid in the hole was drained with the silicone-tipped flute needle and an adequate fluid-air exchange was performed. Gas or sterile air was respectively applied before and after July 2016, and the patients were asked to keep face-down position for 2 or 1 week respectively.

\section{Statistical analysis}

All the clinical features, OCT parameters and surgical outcomes were compared between genders. BCVA was converted to logarithm of minimum angle of resolution (Log MAR) units. Statistical analysis was performed by SPSS 24.0 statistical software program (SPSS for Windows, Chicago, IL, USA). T-test was used for continuous variables when they obeyed normal distribution and Mann-Whitney U test was used when they did not. Pearson's chi-square test and Fisher's exact test were used for categorical variables. A $P$ value less than 0.05 was considered to be statistically significant. 


\section{Results}

A total of 298 eyes from 280 patients (51 males and 229 females) with IMH of stage 3 (192 eyes) or stage 4 (106 eyes) were enrolled. The average age of all the patients was $64.7 \pm 5.2$ years old. The IMHs exhibited an average hole size of $(620.5 \pm 137.6) \mu \mathrm{m}$ in MLD. The general primary closure rate was $92.3 \%$ (275/298), and the mean BCVA improved from 0.12 (Snellen 20/167) to 0.52 (Snellen 20/38) during a mean follow-up of $(6.67 \pm 4.33)$ months.

Comparing IMHs between genders of all the preoperative characteristics, women exhibited a significantly higher ratio of stage3/stage4 $(P=0.045$, Pearson's chisquare test), higher IOP of the affected eye $(P=0.022$, Mann-Whitney U test), and shorter AXL of both eyes $(P=0.001$ for the affected eye, t-test; $P<0.001$ for the fellow eye, Mann-Whitney U test) than men (Table 1), while other characteristics like age of onset and duration of symptoms showed no significant differences. For morphological parameters by OCT, women exhibited larger $\operatorname{MLD}(P=0.009$, t-test $)$ in the horizontal orientation, but no difference in the vertical direction compared to men. Meanwhile, women demonstrated smaller $\mathrm{H}, \mathrm{MHI}$, and THI, but larger DHI and HFF in both orientations (Table 2). CMT $(P<0.001$, Mann-Whitney $U$ test) and adjusted CMT $(P=0.045$, Mann-Whitney $U$ test $)$ of the fellow eyes were also significantly thinner in women than in men.

As listed in Table 1, with comparable rate of combined cataract surgery and gas tamponade, the two genders exhibited similar primary closure rate $(96.2 \%$ vs. $91.3 \%$, $P=0.39$, Fisher's exact test) and highest BCVA (Snellen $20 / 36$ vs. $20 / 39, P=0.23$, Mann-Whitney $U$ test) within the comparable follow-up $(P=0.08$, Mann-Whitney $U$ test). However, men demonstrated significantly shorter recovery duration ( $P=0.024$, Mann-Whitney $U$ test). Patients who underwent combined cataract surgery exhibited no significant difference in recovery duration between male and female $(4.88 \pm 4.00$ vs. $7.16 \pm 6.99, P=$ 0.11, Mann-Whitney $U$ test), while in patients who did not undergo combined cataract surgery, the recovery duration of male was significantly shorter than that of female $(3.40 \pm 3.91$ vs. $11.2 \pm 9.60, \quad P=0.044$, MannWhitney U test). Women who did not undergo combined cataract surgery exhibited significantly shorter recovery duration than those who did (7.16 \pm 6.99 vs. 11.2 $\pm 9.60, P=0.048$, Mann-Whitney $U$ test). However, men did not demonstrate the same difference (4.88 \pm 4.00 vs. $3.40 \pm 3.91, P=0.34$, Mann-Whitney $U$ test).

Since the stage constituent ratio differed significantly between genders, comparisons of the main clinical

Table 1 Comparisons of clinical characteristics between genders

\begin{tabular}{|c|c|c|c|}
\hline & Male & Female & $\mathbf{P}$ \\
\hline No., patients/eyes & $51 / 55$ & $229 / 243$ & / \\
\hline Affected eye, OD/OS & $25 / 30$ & $121 / 122$ & 0.56 \\
\hline Bilaterality, bilateral/unilateral & $4 / 47$ & $14 / 215$ & 0.75 \\
\hline Age of onset, years & $65.2 \pm 5.9$ & $63.8 \pm 5.1$ & 0.17 \\
\hline Duration of symptoms, months & $9.65 \pm 1.42$ & $9.29 \pm 0.68$ & 0.51 \\
\hline Stage, stage3/stage4 & $29 / 26$ & $163 / 80$ & $0.045 *$ \\
\hline \multicolumn{4}{|l|}{ Pre-operative BCVA, Log MAR (Snellen) } \\
\hline Affected eye & $1.03 \pm 0.33(20 / 167)$ & $1.08 \pm 0.39(20 / 167)$ & 0.51 \\
\hline Fellow eye & $0.21 \pm 0.34(20 / 27)$ & $0.19 \pm 0.29(20 / 27)$ & 0.74 \\
\hline \multicolumn{4}{|l|}{ IOP, mmHg } \\
\hline Affected eye & $14.5 \pm 3.0$ & $15.6 \pm 3.2$ & $0.022 *$ \\
\hline Fellow eye & $15.1 \pm 3.4$ & $15.3 \pm 3.1$ & 0.71 \\
\hline \multicolumn{4}{|l|}{$\mathrm{AXL}, \mathrm{mm}$} \\
\hline Affected eye & $23.6 \pm 0.7$ & $23.2 \pm 0.9$ & $0.001 *$ \\
\hline Fellow eye & $23.7 \pm 0.8$ & $23.2 \pm 1.0$ & $<0.001^{*}$ \\
\hline Combined cataract surgery, Yes/No & $47 / 6$ & $212 / 19$ & 0.47 \\
\hline Tamponade, air/gas & $35 / 18$ & $133 / 98$ & 0.26 \\
\hline Follow-up, months & $5.63 \pm 4.47$ & $8.00 \pm 7.07$ & 0.08 \\
\hline Primary closure rate & $96.2 \%$ & $91.3 \%$ & 0.39 \\
\hline Highest post-operative BCVA, Log MAR (Snellen) & $0.34 \pm 0.34(20 / 36)$ & $0.38 \pm 0.33(20 / 39)$ & 0.23 \\
\hline Recovery duration, months & $4.72 \pm 3.98$ & $7.52 \pm 7.33$ & $0.024 *$ \\
\hline
\end{tabular}

*, $P<0.05 ; B C V A$ Best-corrected visual acuity, Log MAR Logarithm of minimum angle of resolution, IOP Intraocular pressure, $A X L$ Axial length 
Table 2 Comparisons of OCT parameters between genders

\begin{tabular}{|c|c|c|c|}
\hline & Male & Female & $\mathbf{P}$ \\
\hline \multicolumn{4}{|l|}{ Horizontal } \\
\hline$M L D, \mu m$ & $577.1 \pm 135.9$ & $630.5 \pm 136.3$ & $0.009 *$ \\
\hline $\mathrm{BD}, \mu \mathrm{m}$ & $1184.7 \pm 233.8$ & $1166.3 \pm 210.6$ & 0.94 \\
\hline $\mathrm{H}, \mu \mathrm{m}$ & $489.1 \pm 84.6$ & $458.3 \pm 53.2$ & $0.028 *$ \\
\hline $\mathrm{FC}, \mu \mathrm{m}$ & $607.6 \pm 180.4$ & $535.9 \pm 142.7$ & 0.08 \\
\hline $\mathrm{DHI}$ & $0.49 \pm 0.09$ & $0.54 \pm 0.08$ & $<0.001 *$ \\
\hline $\mathrm{MHI}$ & $0.42 \pm 0.07$ & $0.40 \pm 0.08$ & $0.042 *$ \\
\hline THI & $0.90 \pm 0.30$ & $0.76 \pm 0.21$ & $0.001 *$ \\
\hline HFF & $1.49 \pm 0.09$ & $1.54 \pm 0.08$ & $<0.001 *$ \\
\hline \multicolumn{4}{|l|}{ Vertical } \\
\hline$M L D, \mu m$ & $541.1 \pm 132.9$ & $578.5 \pm 141.4$ & 0.07 \\
\hline $\mathrm{BD}, \mu \mathrm{m}$ & $1089.8 \pm 201.5$ & $1088.1 \pm 205.9$ & 0.95 \\
\hline $\mathrm{H}, \mu \mathrm{m}$ & $485.4 \pm 79.6$ & $454.5 \pm 52.7$ & $0.012 *$ \\
\hline $\mathrm{FC}, \mu \mathrm{m}$ & $548.7 \pm 168.3$ & $509.5 \pm 136.7$ & 0.07 \\
\hline $\mathrm{DHI}$ & $0.50 \pm 0.11$ & $0.53 \pm 0.09$ & $0.016 *$ \\
\hline $\mathrm{MHI}$ & $0.46 \pm 0.09$ & $0.43 \pm 0.09$ & $0.043 *$ \\
\hline $\mathrm{THI}$ & $0.99 \pm 0.49$ & $0.84 \pm 0.27$ & $0.008 *$ \\
\hline HFF & $1.50 \pm 0.11$ & $1.53 \pm 0.09$ & $0.016 *$ \\
\hline CMT (fellow eye), $\mu \mathrm{m}$ & $247.6 \pm 17.6$ & $234.7 \pm 13.4$ & $<0.001^{*}$ \\
\hline $\begin{array}{l}\text { Adjusted CMT } \\
\text { (CMT/AXL, fellow eye), } \\
\mu \mathrm{m} / \mathrm{mm}\end{array}$ & $10.5 \pm 0.9$ & $10.1 \pm 0.7$ & $0.045 *$ \\
\hline
\end{tabular}

*, $P<0.05 ;$ OCT Optical coherence tomography, MLD Minimum linear diameter, $B D$ Basal diameter, $H$ Height of hole, $F C$ Fluid cuff, $D H I$ Diameter hole index, MHI Macular hole index, THI Traction hole index, HFF Hole form factor, CMT Central macular thickness, AXL Axial length

features and OCT parameters were also conducted within stage respectively (Table 3 ). For IMHs of stage 3, women exhibited significantly younger age of onset $(P=$ 0.023 , Mann-Whitney U test), larger MLD $(P=0.003$ in horizontal, $P=0.015$ in vertical, both Mann-Whitney U test), and smaller $\mathrm{H}(P=0.07$, in horizontal, $P=0.029$ in vertical, both Mann-Whitney $U$ test) than men, while other clinical characteristics and surgical outcomes showed no difference. For IMHs of stage 4, all the pre-, intra-, and post-operative metrics exhibited no significant differences between genders.

\section{Discussion}

According to previous epidemiological investigations [3] and clinical reports [12], IMH is much more common to occur in women than in men. This study enrolled a population with a female to male ratio of about 4:1, which is higher than that of previous epidemiological investigations [3], but comparable to other retrospective clinical reports concerning surgical patients [13, 14]. The data presented here reveal that IMHs of women seem to be larger and occur earlier than those of men, but there are no significant differences between genders concerning other pre-operative metrics and surgical outcomes.

In this study, the stage constituent ratio (stage3/ stage4) of IMHs was significantly higher in women than in men. According to Gass's classification system [9], the difference between IMH of the two stages is mainly the status of the vitreous at the optic disc, so that women owned more IMHs of stage 3 indicated the possibility that complete posterior vitreous detachment (PVD) occurs later in women than in men. The study of Schwab and colleagues observed 335 non-myopic eyes and found that women were significantly older than men when having the late-stage PVD (complete PVD) in the eyes [15], which was consistent with our speculation. This phenomenon could be explained by the findings of van Deemter and colleagues that female vitreous, especially after 50 years of age, experiences a faster accumulation of pentosidine [16], which is associated with the absence of a complete PVD [17].

Kazuyuki et al. observed 526 eyes of 480 patients with stage 3 or stage 4 IMHs that had undergone vitrectomy, and found that females of stage 3 demonstrated younger age of onset and larger size of macular hole [18]. Our results seem to be in agreement with this. However, the report of Kazuyuki et al.'s was not in English and the full text was not available online, so we cannot obtain any further details.

In high myopic eyes, macular holes occur earlier [19] and evolve faster than in non-myopic eyes [20] due to longer axial length [20] and thinner retina thickness [21] because these two factors may exacerbate the impact of traction forces on highly myopic eyes [22].

In the present study, the foveal retina thickness of eyes with IMH was unable to know owing to the hole, so CMT of the fellow eye was measured to represent. The results showed that AXL was longer and CMT was thicker in male than in female, which were in accordance with previous reports $[23,24]$ but seemed to be a contradiction concerning their opposite effects on development of macular holes. Meanwhile, CMT is positively related to AXL [24]. Therefore, we introduced the concept of adjusted CMT calculated by CMT/AXL, regarding CMT and AXL as a whole, and found it significantly smaller in female than in male. This could probably explain why female exhibited younger age of onset in IMHs of stage 3. In other words, though developing stage $3 \mathrm{IMH}$ earlier, women tend to remain in the same stage longer than men, and this also explains why IMHs of stage 4 showed no significant difference between genders in age of onset in our results.

With respect to morphological metrics, female IMHs exhibited larger MLD than male on the whole, which largely attribute to the difference in stage 3 IMHs. According to previous studies, MLD enlarges as the IMH 
Table 3 Comparisons between genders in stage 3 and stage 4 IMHs

\begin{tabular}{|c|c|c|c|c|c|c|}
\hline & \multicolumn{3}{|c|}{ Stage $3(n=192)$} & \multicolumn{3}{|c|}{ Stage $4(n=106)$} \\
\hline & Male $(n=29)$ & Female $(n=163)$ & $\mathbf{P}$ & Male $(n=26)$ & Female $(n=80)$ & $P$ \\
\hline Age of onset, years & $66.7 \pm 6.7$ & $63.6 \pm 5.4$ & $0.023 *$ & $63.7 \pm 4.6$ & $64.7 \pm 4.3$ & 0.30 \\
\hline Duration of symptoms, months & $6.79 \pm 4.10$ & $8.81 \pm 10.10$ & 0.56 & $12.6 \pm 14.0$ & $10.1 \pm 10.7$ & 0.18 \\
\hline Combined cataract surgery, Yes/No & $27 / 2$ & $143 / 13$ & 1.00 & $20 / 4$ & $69 / 6$ & 0.20 \\
\hline Tamponade, air/gas & $19 / 10$ & $90 / 66$ & 0.43 & $16 / 8$ & $43 / 32$ & 0.42 \\
\hline Follow-up, months & $5.27 \pm 3.85$ & $6.84 \pm 4.30$ & 0.11 & $6.10 \pm 5.23$ & $7.11 \pm 4.23$ & 0.24 \\
\hline Primary closure rate & $96.6 \%$ & $91.0 \%$ & 0.47 & $95.8 \%$ & $92.0 \%$ & 1.00 \\
\hline Recovery duration, months & $4.62 \pm 4.00$ & $7.41 \pm 7.29$ & 0.07 & $4.86 \pm 4.04$ & $7.75 \pm 7.46$ & 0.17 \\
\hline \multicolumn{7}{|l|}{ Horizontal } \\
\hline$M L D, \mu m$ & $563.5 \pm 136.1$ & $630.6 \pm 126.1$ & $0.003 *$ & $592.4 \pm 136.6$ & $629.9 \pm 156.1$ & 0.28 \\
\hline $\mathrm{BD}, \mu \mathrm{m}$ & $1176.7 \pm 248.0$ & $1174.4 \pm 204.4$ & 0.49 & $1193.6 \pm 221.5$ & $1148.9 \pm 223.3$ & 0.38 \\
\hline $\mathrm{H}, \mu \mathrm{m}$ & $487.8 \pm 80.4$ & $457.3 \pm 52.9$ & 0.07 & $490.5 \pm 90.6$ & $459.7 \pm 54.0$ & 0.11 \\
\hline \multicolumn{7}{|l|}{ Vertical } \\
\hline MLD, $\mu \mathrm{m}$ & $520.3 \pm 121.9$ & $580.9 \pm 136.1$ & $0.015 *$ & $564.2 \pm 143.0$ & $573.7 \pm 152.4$ & 0.78 \\
\hline $\mathrm{BD}, \mu \mathrm{m}$ & $1083.3 \pm 210.1$ & $1093.0 \pm 200.1$ & 0.81 & $1097.0 \pm 195.3$ & $1077.9 \pm 218.2$ & 0.69 \\
\hline $\mathrm{H}, \mu \mathrm{m}$ & $485.6 \pm 74.9$ & $452.4 \pm 52.0$ & $0.029 *$ & $485.2 \pm 86.0$ & $458.7 \pm 54.4$ & 0.15 \\
\hline
\end{tabular}

*, $P<0.05$; IMH Idiopathic macular hole, MLD Minimum linear diameter, $B D$ Basal diameter, $H$ Height of hole

progresses, while enlargement of $\mathrm{BD}$ slows down [25]. Since female develop stage $3 \mathrm{IMHs}$ earlier and stay in the same stage longer, it is reasonable that female IMHs of stage 3 are larger in MLD than male. As the disease evolves, the difference of MLD became much less prominent between genders in stage 4 IMHs, which indicates that IMHs of both male and female evolve into a similar ultimate state.

The height of the hole was smaller in women than in men. Previous reports gave different conclusions on whether height of macular hole could predict surgical outcomes [26, 27]; however, their definition of the height - the greatest distance between the RPE layer and the vitreoretinal interface- differed from ours. In the present study, height of the hole was defined as the vertical height of the neuroepithelia edge starting from the junction point of the detached photoreceptor and the RPE, which we believe to be in accordance more with retinal thickness than retinal edema. In this regard, smaller $\mathrm{H}$ represents a relatively thinner foveal retina, which is consistent with the normal variation that CMT is smaller in women than in men.

Other derived indexes were calculated by MLD, BD, and $\mathrm{H}$, so they showed understandable differences between genders in accordance with the three primary parameters.

According to previous studies, primary closure rate is mainly correlated with MLD and duration of symptoms, and post-operative BCVA with MLD and times of surgery [28]. In this study, men and women showed similar primary closure rate and comparable highest BCVA during follow-up. That is to say, the differences between genders mentioned above may largely attribute to normal variations in foveal anatomy and physiology, but demonstrate limited influence on surgical outcomes; in other words, female IMHs may be not more severe than male as it may be anticipated. Our results showed that women who did not undergo combined cataract surgery demonstrated significantly longer recovery duration, which resulted in that women exhibited longer recovery duration than men in general. The larger MLD and the thinner adjusted CMT of female could be one of the causes, but further elucidation is in need. Besides, the ellipsoid zone and the external limiting membrane could regenerate slower in eyes with larger MLD, which may also affect the recovery duration.

This retrospective study enrolled only patients who underwent operation, which may lead to a sample selection bias; however, the relatively large size of sample with rational gender proportion and rigorous methods could offer reliable results to a large extent. Usually, stage 2 IMHs are asymmetric in most scan directions because of the vitreomacular traction, which may lead to inaccuracy of parameter measurements like MLD and $\mathrm{H}$. Thus, IMHs of stage 2 were excluded in this study.

\section{Conclusion}

This study comprehensively compares the genderrelated differences in IMHs of stage 3 and stage 4 with a relatively large size of sample, which may enrich perception of IMHs and provide references for further research. Though women seem to develop IMHs earlier 
and larger, these differences could be explained by the normal anatomical and physiological variations between genders to a certain extent, and may not considerably influence the surgical outcomes.

\section{Abbreviations}

IMH: Idiopathic macular hole; CSC: Central serous chorioretinopathy; IOP: Intraocular pressure; AXL: Axial length; BCVA: Best-corrected visual acuity; MLD: Minimum linear diameter; RPE: Retinal pigment epithelium; BD: Basal diameter; H: Height; FC: Fluid cuff; DHI: Diameter hole index; MHI: Macular hole index; THI: Traction hole index; HFF: Hole form factor; CMT: Central macular thickness; ILM: Internal limiting membrane; Log MAR: Logarithm of minimum angle of resolution; PVD: Posterior vitreous detachment

\section{Acknowledgements}

Not applicable.

\section{Authors' contributions}

JW, YY and WL conceived and designed research; JW and YY collected data and conducted research; All authors were involved in the analysis; JW and YY wrote the initial paper; $W L, X L, Z W$ and $B Q$ revised the paper; $W L$ had primary responsibility for final content. All authors read and approved the final manuscript.

\section{Funding}

No funding was involved in this research.

\section{Availability of data and materials}

All data relevant to the study are included in the article. The deidentified participant data are in the team's database, which is available only from the corresponding author. For reusing, please contact WL (wuliubj@sina.com) for permission.

\section{Ethics approval and consent to participate}

The study was conducted in accordance with the tenets of Declaration of Helsinki. Access of the data used in this research was permitted by the Ethics Committee of Beijing Tongren Hospital (TRECKY2019-097). All of the participants gave written informed consent after sufficient explanation of the whole research protocal.

\section{Consent for publication}

Not applicable.

\section{Competing interests}

The authors declare that they have no competing interests.

Received: 14 March 2020 Accepted: 1 September 2020

Published online: 10 September 2020

\section{References}

1. Ezra E. Idiopathic full-thickness macular hole: natural history and pathogenesis. Br J Ophthalmol. 2001;85:102-8.

2. Ali FS, Stein JD, Blachley TS, et al. Incidence of and risk factors for developing idiopathic macular hole among a diverse Group of Patients throughout the United States. JAMA Ophthalmol. 2017;135:299-305.

3. McCannel CA, Ensminger JL, Diehl NN, et al. Population-based incidence of macular holes. Ophthalmology. 2009;116:1366-9.

4. Jackson $\mathrm{TL}$, Nicod E, Simpson A, et al. Symptomatic vitreomacular adhesion. Retina. 2013;33:1503-11.

5. Chatziralli I, Kabanarou SA, Parikakis E, et al. Risk factors for central serous Chorioretinopathy: multivariate approach in a case-control study. Curr Eye Res. 2017;42:1069-73.

6. Hanumunthadu D, Van Dijk EHC, Gangakhedkar S, et al. Gender variation in central serous chorioretinopathy. Eye (Lond). 2018;32:1703-9.

7. Rudnicka AR, Jarrar Z, Wormald R, et al. Age and gender variations in agerelated macular degeneration prevalence in populations of European ancestry: a meta-analysis. Ophthalmology. 2012;119:571-80.

8. Wagner-Schuman M, Dubis AM, Nordgren RN, et al. Race- and sex-related differences in retinal thickness and foveal pit morphology. Invest Ophthalmol Vis Sci. 2011;52:625-34.
9. GASS JD. Reappraisal of biomicroscopic classification of stages of development of a macular hole. Am J Ophthalmol. 1995;119:752-9.

10. Duker JS, Kaiser PK, Binder S, et al. The international Vitreomacular traction study group classification of vitreomacular adhesion, traction, and macular hole. Ophthalmology. 2013;120:2611-9.

11. Mo B, Liu W. Macular thickness measurements with spectral domain optical coherence tomography in normal younger eyes. Ophthalmol CHN. 2011;20: 255-8.

12. Wakely L, Rahman R, Stephenson J. A comparison of several methods of macular hole measurement using optical coherence tomography, and their value in predicting anatomical and visual outcomes. Br J Ophthalmol. 2012; 96:1003-7.

13. Kang SW, Ahn K, Ham DI. Types of macular hole closure and their clinical implications. Br J Ophthalmol. 2003;87:1015-9.

14. He F, Dong F, Yu W, et al. Recovery of photoreceptor layer on spectraldomain optical coherence tomography after vitreous surgery combined with air tamponade in chronic idiopathic macular hole. Ophthalmic Surg Lasers Imaging Retina. 2015;46:44-8.

15. Schwab C, Ivastinovic D, Borkenstein A, et al. Prevalence of early and late stages of physiologic PVD in emmetropic elderly population. Acta Ophthalmol. 2012;90:179-84.

16. van Deemter $\mathrm{M}$, Ponsioen $\mathrm{TL}$, Bank RA, et al. Pentosidine accumulates in the aging vitreous body: a gender effect. Exp Eye Res. 2009;88:1043-50.

17. van Deemter M, Bank RA, Vehof J, et al. Factors associated with pentosidine accumulation in the human vitreous. Retina. 2017;37:770-7.

18. Kumagai K. Clinical features of idiopathic macular holes-differences between sexes and stages. Jpn J Ophthalmol. 2002;46:118.

19. Kobayashi H, Kobayashi K, Okinami S. Macular hole and myopic refraction. Br J Ophthalmol. 2002;86:1269-73.

20. Ruiz-Medrano J, Montero JA, Flores-Moreno I, et al. Myopic maculopathy: current status and proposal for a new classification and grading system (ATN). Prog Retin Eye Res. 2018;69:80-115.

21. Wu TT, Kung YH. Comparison of anatomical and visual outcomes of macular hole surgery in patients with high myopia vs. non-high myopia: a case-control study using optical coherence tomography. Graefes Arch Clin Exp Ophthalmol. 2012;250:327-31.

22. Chuang LH, Chen YP, Wang NK, et al. Macular hole repair by Vitrectomy and internal limiting membrane peeling in highly myopic eyes. Retina. 2014;34: 2021-7.

23. Li M, Cai Y, Pan R, et al. The study of anterior sclera thickness and associated ocular parameters. Chin J Exp Ophthalmol. 2013;31:81-5.

24. Jonas JB, Xu L, Wei WB, et al. Retinal thickness and axial length. Invest Ophthalmol Vis Sci. 2016:57:1791-7.

25. Yun C, Oh J, Hwang SY, et al. Morphologic characteristics of chronic macular hole on optical coherence tomography. Retina. 2012;32:2077-84.

26. Ruiz-Moreno JM, Staicu C, Piñero DP, et al. Optical coherence tomography predictive factors for macular hole surgery outcome. Br J Ophthalmol. 2008; 92:640-4.

27. Kusuhara S, Negi A. Predicting visual outcome following surgery for idiopathic macular holes. Ophthalmologica. 2014;231:125-32.

28. American Academy of Ophthalmology Retina/Vitreous Panel. Preferred practice pattern ${ }^{\circledast}$ guidelines. Idiopathic macular hole. San Francisco: American Academy of Ophthalmology; 2014.

\section{Publisher's Note}

Springer Nature remains neutral with regard to jurisdictional claims in published maps and institutional affiliations. 